\title{
Diagnóstico de enfermagem Risco de Suicídio em idosos: revisão integrativa
}

\author{
Nursing diagnosis for Risk of Suicide in elderly: integrative review \\ Diagnóstico de enfermería Riesgo de Suicidio en ancianos: revisión integrativa
}

\author{
Girliani Silva de Sousa ${ }^{a}$ \\ Jaqueline Galdino Albuquerque Perrellib \\ Everton Sougey Botelho ${ }^{\mathrm{a}}$
}

\section{Como citar este artigo:}

Sousa GS, Perrelli JGA, Botelho ES.

Diagnóstico de enfermagem Risco de

Suicídio em idosos: revisão integrativa.

Rev Gaúcha Enferm. 2018;39:e2017-

0120. doi: https://doi.org/10.1590/1983-

1447.2018.2017-0120. a Universidade Federal de Pernambuco (UFPE), Programa de Pós-Graduação em Neuropsiquiatria e Ciências do Comportamento. Recife, Pernambuco, Brasil.

' Universidade Federal de Pernambuco (UFPE), Graduação em Enfermagem, Campus Vitória de Santo Antão. Vitória de Santo Antão, Pernambuco, Brasil.

\section{RESUMO}

Objetivo: Analisar a literatura específica acerca dos fatores de risco do diagnóstico de enfermagem Risco de Suicídio de idosos. Método: Trata-se de uma revisão integrativa da literatura realizada em junho de 2015 nas bases de dados MEDLINE, PsycINF0 e CINAHL, sem limite temporal.

Resultados: Foram analisados 80 artigos. Sugere-se a inclusão de 23 fatores de risco na taxonomia da NANDA-I: apatia; agitação; baixa autoestima; descuido com a medicação; incapacidade de pedir ajuda; incapacidade de expressar sentimentos; plano suicida; rigidez; incapacidade funcional; problemas visuais; tristeza; hostilidade; ansiedade; fracasso; frustração; infelicidade; desonra; frequentes visitas a médico com sintomas vagos; privação social; desvalorização social; violência psicológica; violência intrafamiliar e violência financeira.

Conclusão: 0 risco de suicídio apresentado na taxonomia da NANDA - I necessita de refinamento e adaptação ao contexto de idosos, sobretudo para averiguação dos fatores de risco que não estão contidos na referida classificação.

Palavras-chave: Diagnóstico de enfermagem. Suicídio. Idoso. Revisão.

\section{ABSTRACT}

Objective: To analyze the specialized literature regarding the risk factors in nursing diagnosis for suicide risks in elderly.

Method: This is an integrative literature review carried out during June 2015 in the following databases with no time limit: MEDLINE, PsycINFO and CINAHL.

Results: A total of 80 full papers were analyzed. It was suggested the inclusion of 23 risk factors in NANDA-I taxonomy: apathy; unrest; low self esteem; carelessness with medication; Inability to ask for help; Inability to express feelings; suicidal plan; rigidity; functional disability; visual problems; sadness; hostility; anxiety; failure; frustration; unhappiness; dishonor; frequent visits to a physician with unclear symptoms; social deprivation; social devaluation; psychological violence; Interfamilial violence; and financial violence.

Conclusion: The risks for suicide presented in NANDA-I taxonomy need to be refined and adapted to the elderly reality. Furthermore, a review is also recommended for the risk factors not included in this classification.

Keywords: Nursing diagnosis. Suicide. Aged. Review.

\section{RESUMEN}

Objetivo: Analizar la literatura específica acerca de los factores de riesgo en el diagnóstico de enfermería sobre los riesgos de suicidio en ancianos.

Método: Revisión completa en la literatura, realizada en junio del 2015, sobre la base de datos MEDLINE, PsycINFO y CINAHL, sin límite de tiempo.

Resultados: Se analizaron 80 artículos. Se sugiere la introducción de 23 factores de riesgo en la Taxonomía de NANDA-l: apatía, agitación, autoestima baja, descuido con la medicación, incapacidad de pedir ayuda, incapacidad de expresar sentimientos, pensamientos suicidas, incapacidad funcional, problemas visuales, tristeza, hostilidad, ansiedad, fracaso, frustración, infelicidad, deshonor, visitas frecuentes al médico con síntomas vagos, comportamientos antisociales, violencia psicológica, actitudes violentas dentro del seno familiar.

Conclusión: El riesgo de suicidio presente en la taxonomía de NANDA-I necesita de mejorías y adaptación para ser aplicado en ancianos, sobre todo para aclarar los factores de riesgo que no se encuentran en esta clasificación.

Palabras clave: Diagnóstico de enfermería. Suicidio. Anciano. Revisión 


\section{INTRODUÇÃO}

O suicídio é um grave problema de saúde pública, sobretudo na população idosa que aumenta de forma exponencial em nível mundial(1-2). A Organização Mundial de Saúde (OMS) estima que as taxas de suicídio sejam mais elevadas em pessoas com idade acima de 70 anos em quase todas as regiões do planeta(3). Fato que chama atenção é a estreita relação entre tentativas e efetivação do suicídio: entre os jovens, essa relação é de 100 a 200 tentativas para uma morte consumada; na população idosa, a cada duas a três pessoas tentando o ato sem êxito, ocorre um óbito ${ }^{(4-5)}$.

No Rio Grande do Sul, Brasil, encontrou-se que 15,9\% dos idosos estavam em risco de suicídio( ${ }^{(6)}$. O suicídio de idosos é mais planejado e deliberado do que em outras faixas etárias ${ }^{(7)}$, o que reforça a necessidade do enfermeiro ter conhecimento acerca dos fatores relacionados com o diagnóstico de enfermagem (DE) Risco de suicídio nessa população para identificar, compreender e tratar as vulnerabilidades para esse fenômeno.

Nesse contexto, a taxonomia da NANDA - I apresenta o fenômeno Risco de suicídio composto por 47 fatores de risco e definido como vulnerabilidade à lesão autoinfligida que ameaça a vida ${ }^{(8)}$. Acredita-se que esses fatores de risco sugeridos pela NANDA-I para o referido diagnóstico necessitam ser refinados, uma vez que alguns fatores apresentam relação com o risco de suicídio ${ }^{(1-2)}$ e, no entanto, não constam na taxonomia ${ }^{(8)}$. Dessa forma, objetivou-se analisar a literatura específica acerca dos fatores de risco do diagnóstico de enfermagem Risco de Suicídio de idosos.

\section{MÉTODO}

Trata-se de uma revisão integrativa da literatura elaborada a partir da proposta de Ganong ${ }^{(9)}$. A primeira fase, referente à pergunta condutora, teve o seguinte questionamento: quais os fatores associados ao diagnóstico de enfermagem Risco de suicídio em idosos? Posteriormente, seguiu-se para a segunda fase denominada: seleção dos estudos.

A busca dos artigos ocorreu nas bases de dados: Medline (Literatura Internacional em Ciências da Saúde), Psycinfo (Behavioral and Social Science Research) e Cinahl (Cummulative Index to Nursing and Allied Health Literature). Utilizaram-se os seguintes descritores: suicídio, idosos e enfermagem. Utilizaram-se duas estratégias de busca com os descritores selecionados: a primeira os cruzou utilizando o ícone AND e na segunda estratégia os cruzamentos foram realizados por meio do ícone OR. O levantamento dos estudos ocorreu no mês de junho de 2015. Adotaram-se os seguintes critérios de inclusão: artigos que respondam à temática, publicados em português, espanhol ou inglês, sem limite temporal e disponíveis integralmente online.

A pesquisa nas bases de dados resultou em 2.624 artigos, os quais passaram por uma pré-seleção por meio da leitura dos títulos e dos resumos. Ao término dessa fase foram pré-selecionados 119 artigos que foram lidos na íntegra, resultando em 80 artigos. Os motivos da exclusão de 2.544 estudos encontram-se descritos na Tabela 1.

Tabela 1 - Descrição dos motivos de exclusão dos artigos

\section{Motivo de exclusão do artigo}

n

Indisponíveis na íntegra

Cartas editoriais e anais de evento

20

29

Prevenção do suicídio

241

Artigos redigidos nos idiomas francês, alemão e japonês

279

Epidemiologia do suicídio na população em geral

313

Idosos institucionalizados

323

Autonegligência, quedas e homicídios

330

Ideação e tentativa de suicídio

920

\section{Total de artigos que não atenderam os critérios de inclusão}

2.544

Fonte: Dados da pesquisa, 2015.

A terceira fase correspondeu à caracterização dos estudos. Para isso, extraíram-se dos artigos as informações: (1) Dados gerais sobre o artigo; (2) Características metodo- lógicas e (3) Descrição dos fatores de risco do DE Risco de suicídio em idosos. A quarta e a quinta fase dizem respeito à análise dos dados primários dos estudos incluídos na re- 
visão integrativa e à interpretação dos dados, evidenciando as singularidades e as peculiaridades dos artigos acerca do tema central. A sexta fase corresponde a sumarização da revisão integrativa articulando aos fatores que levaram os idosos a morrer por suicídio. Nesta revisão, a qualidade dos estudos foi avaliada de acordo com a classificação do nível de evidência das pesquisas de Melnyk, Fineout-Overholt(10) (Quadro 1).

\begin{tabular}{|c|l|}
\hline Nível & \\
\hline I & $\begin{array}{l}\text { Evidências oriundas de relevantes revisões sistemáticas ou meta-análises de todos os ensaios clínicos } \\
\text { randomizados controlados ou provenientes de diretrizes clínicas baseadas em revisões sistemáticas de } \\
\text { ensaios clínicos randomizados controlados }\end{array}$ \\
\hline II & Evidências derivadas de pelo menos um ensaio clínico randomizado controlado bem delineado \\
\hline III & Evidências obtidas de ensaios clínicos bem delineados sem randomização \\
\hline IV & Evidências provenientes de estudos de coorte e de caso-controle bem delineados \\
\hline$V$ & Evidências originárias de revisão sistemática de estudos descritivos e qualitativos \\
\hline$V I$ & Evidências derivadas de um único estudo descritivo ou qualitativo \\
\hline VII & Evidências oriundas de opinião de autoridades e/ou relatórios de comitês de especialistas. \\
\hline
\end{tabular}

Quadro 1 - Classificação dos níveis de evidência

Fonte: Melnyk, Fineout-Overholt(10)

Concomitantemente, após leitura exaustiva dos artigos incluídos na presente revisão, articularam-se os dados evidenciados nos resultados das pesquisas, o conhecimento teórico nesta temática e as implicações para a prática clínica da enfermagem. A partir da discussão e interpretação dos resultados, argumenta-se com base na literatura a inclusão de novos fatores de risco do DE Risco de suicídio em idosos indexados na taxonomia da NANDA-I. A última etapa corresponde à síntese do conhecimento evidenciado nos artigos analisados. Dessa forma, as evidências dos fatores de risco foram agrupadas quanto aos aspectos psicológicos, demográficos, físicos, emocionais e situacionais.

\section{Q RESULTADOS}

A distribuição geográfica dos artigos seleciona$\operatorname{dos}^{(1-2,4,6,11-85)}$ concentrou-se na Europa (47\%), Estados Unidos (29,3\%) e Ásia (15\%). Destaca-se que 7,5\% foram desenvolvidos no Brasil. No tocante à área de publicação, $52,7 \%$ concentraram-se na psiquiatria, 39,9\% na geriatria (39,9\%) e 7,4\% na saúde pública. Nos últimos cinco anos, obteve-se um número expressivo de publicações (39,2\%).

Sobre a classificação do nível de evidência dos estudos e seus desenhos metodológicos, 61,8\% possuem o nível IV, ou seja, são estudos de coorte e de caso-controle, seguido de 22,3\% com nível VI, que corresponde a único estudo descritivo ou qualitativo, e 15,9\% apresentam nível V, caracterizado por revisão sistemática de estudos descritivos e qualitativos.
Sobre os fatores de risco psicológicos (tabela 2), sugere-se o acréscimo de novos fatores de risco à taxonomia da NANDA I: apatia, agitação, baixa autoestima, descuido com a medicação, incapacidade de pedir ajuda, incapacidade de expressar sentimentos, plano suicida e rigidez comportamental. Ademais, sugere-se a alteração de três fatores já existentes: de recuperação eufórica de uma depressão profunda para súbita recuperação de uma depressão profunda; de transtorno psiquiátrico para transtorno mental; e a junção de ameaças de matar-se e verbalização do desejo de morrer para ideação suicida.

Com relação aos de risco físicos contidos na tabela 3, sugere-se o acréscimo da incapacidade funcional e dos problemas visuais na NANDA I, assim como a utilização das expressões doenças crônico-degenerativas e doenças terminais para referir-se, respectivamente, às enfermidades físicas e enfermidades terminais.

Quanto aos sentimentos vivenciados pelos idosos que efetivaram o suicídio (Tabela 4), salienta-se que os fatores tristeza, hostilidade, ansiedade, fracasso, frustração, infelicidade e desonra não estão presentes na NANDA-I. Dessa forma, sugere-se a inclusão dos mesmos na taxonomia.

Sobre os fatores de risco situacionais (Tabela 5), a NANDA I os divide em: situacionais e de risco sociais. Sugere-se a utilização de uma única nomenclatura: fatores de risco situacionais. Ademais, não constam na referida taxonomia: frequentes visitas a médico com sintomas vagos, privação social, desvalorização social, violência psicológica, violência intrafamiliar, violência financeira e autonegligência. 
Tabela 2 - Fatores de risco psicológicos encontrados nas publicações revisadas

\section{Fatores psicológicos}

Depressão(1-2,4,6-7,11-61,80)

Transtorno mental ${ }^{(1-3,3,1,14-19,21-22,24,28-29,3-34,37,39,41,43,45,47-48,59-64,66-68)}$

Ideação suicida (11,4, $12,14,25,29,34-35,37,41-43,45-46,48,51-54,59-61,65,69-72,78-79)$

História de tentativa de suicídio ${ }^{(1,4,6,15,19,22,25,38,44,47,49,53-5,62-63,66,69-71,74-76)}$

Incapacidade de pedir ajuda ${ }^{(1-2,12-14,19,22,35,39,41,50,73-78)}$

Rigidez comportamental(1-2,7,12,22-25,37,43,59,62,76,78)

Doenças psicóticas primárias (esquizofrenia e doença esquizoafetiva) $)^{(1,4,16,19,23,25,28,33,3,3,39,43,47,53)}$

Incapacidade de expressar sentimentos $(2,12-14,22,37,45,52,75,78-79)$

Transtorno bipolar ${ }^{(1,6,17-18,23,28,37,43,45)}$

Plano suicida ${ }^{(1,4,48,53-54,55,69-70)}$

Abuso infanti|(53,55,67,76,78-79,81,85)

Baixa autoestima ${ }^{(1,4,7,24,43,45,64)}$

Descuido com a medicação $0^{(1,48,53,62,66)}$

Transtorno de personalidade $e^{(4,16,34,42)}$

Apatia ${ }^{(1,41,43,52)}$

Armazenamento de medicamentos ${ }^{(37,43,62,60)}$

Fazer testamento ${ }^{(2,53,54,63)}$

Agitação $0^{(1-2,48)}$

Mudança acentuada de atitude $e^{(1,48)}$

Súbita recuperação em relação a um quadro de depressão profunda ${ }^{(1)}$ n

55

68,5

$37 \quad 46,2$

$27 \quad 33,7$

$20 \quad 25,0$

$14 \quad 17,5$

$12 \quad 15,0$

$11 \quad 13,7$

$11 \quad 13,7$

$9 \quad 11,2$

$8 \quad 10,0$

$8 \quad 10,0$

$7 \quad 8,7$

$5 \quad 6,2$

$4 \quad 5,0$

$4 \quad 5,0$

$4 \quad 5,0$

$4 \quad 5,0$

$3 \quad 3,7$

$2 \quad 2,5$

$1 \quad 1,2$

Fonte: Dados da pesquisa, 2015

Tabela 3 - Fatores de risco físicos e demográficos encontrados nas publicações revisadas

\begin{tabular}{|c|c|c|}
\hline Fatores de risco físicos & $\mathbf{n}$ & $\%$ \\
\hline Doenças crônico-degenerativas ${ }^{(1-3,36-7,11-13,19-21,22,24-27,31-33,36-39,41,43-46,48,50,58-60,62-65,67,69,71,73,76-82)}$ & 49 & 61,2 \\
\hline Incapacidade funcional| $\left.\right|^{(1-2,4,7,711,13,22,24-27,29,31,36-37,39,41,43,45-48,52-53,59-60,69,73,76,78-79)}$ & 28 & 35,0 \\
\hline Doenças terminais ${ }^{(1,7,21-22,25-26,29-31,37,41,43,45-48,52-54,58,62,69,78-79)}$ & 23 & 28,7 \\
\hline Dor crônica ${ }^{(1,7,13,22,24,28,32,37,41,43,45-48,53,69,76,78-79)}$ & 18 & 22,5 \\
\hline Problemas visuais (1,13,37,41,46,82) $^{2}$ & 6 & 7,5 \\
\hline Fatores de risco demográficos & $\mathbf{n}$ & $\%$ \\
\hline Sexo masculino $(1-2,13,19-22,24-27,29,32-34,37-39,42,45,49,54,57,60,62,64,67,69,78-81)$ & 28 & 35,0 \\
\hline Viuvez $^{(1,4,7,19,22-25,29,36-38,39,42-43,45-46,49,51-55,58,60,62-63,69,72)}$ & 26 & 32,5 \\
\hline Divórcio (1,4,6,7,21,24-27,37,39,42-43,45-47,53-55,60-63,65,68-69,71,72) & 25 & 31,2 \\
\hline
\end{tabular}

Fonte: Dados da pesquisa, 2015

Tabela 4 - Fatores de risco relacionados às emoções encontradas nas publicações revisadas

\section{Fatores de risco relacionados às emoções}

Solidão(1-2,11,13,20,24,26-28,29-31,37,39,41,43,45-46,48,49,52,55,67,71,76,78-79,83)

Desesperança ${ }^{(1-2,4,11,13,27,30,35,37,41-43,52-53,55,60,62,74-76,78,80)}$

Tristeza ${ }^{(1,13,14,24,29,41,43,45-46,48,52-55,64,71,74,78-80)}$ n $\%$

$22 \quad 27,5$

20
27,5

25,0 


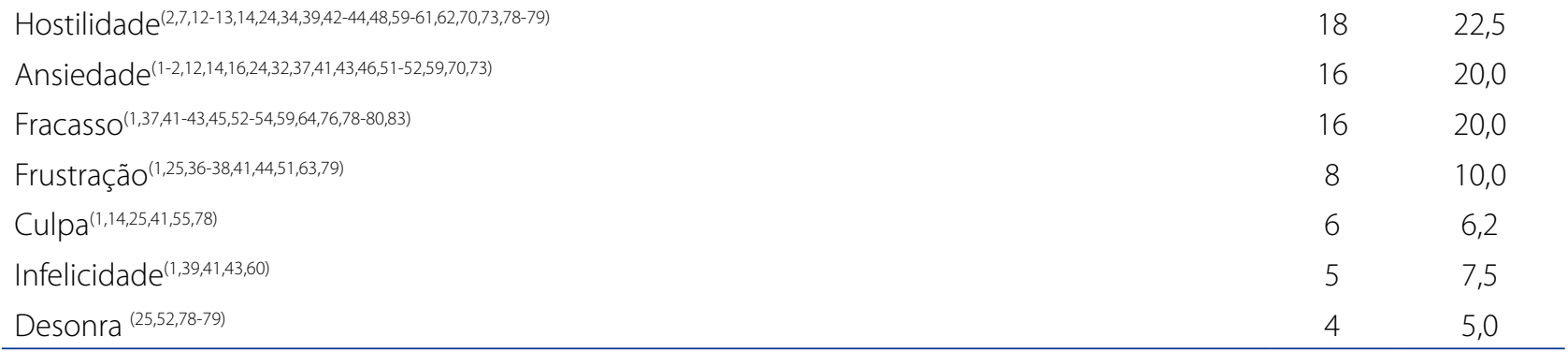

Fonte: Dados da pesquisa, 2015.

Tabela 5 - Fatores de risco situacionais encontrados nas publicações revisadas

\begin{tabular}{|c|c|c|}
\hline Fatores de risco situacionais & $\mathbf{n}$ & $\%$ \\
\hline Abuso de álcoo|(1,4,7,13-15,17-19,21,23-26,31-33,37,40-41,43,45-46,48,51,60-61,67-68,73,75,81) & 33 & 41,2 \\
\hline Conflito Familiar ${ }^{(1-3,7,21,22,27,29,36-39,43,45-46,48,51,53,63,65,71-73,76-77,81)}$ & 27 & 33,7 \\
\hline Apoio social insuficiente ${ }^{(1-3,7,13,22,26-28,35-37,43,45-47,48,52-54,62-63,65,71,73,7,81-83)}$ & 26 & 32,5 \\
\hline Problemas financeiros $(1-2,13,21,22-25,27,36,38,45-46,48,51,53,63-64,71-73,77-78,80-81,85)$ & 25 & 31,2 \\
\hline Dependência funcional $\left.\right|^{(1-3,7,73,21,22-27,29,36-38,43,45-47,53,59,68-69,71,73,76,78)}$ & 24 & 30 \\
\hline Frequentes visitas ao médico com sintomas vagos $(1,7,13-14,27-28,31,33,37,41,48,56-57,61,69,71,80-82,85)$ & 19 & 23,7 \\
\hline Morar só(1-3,6,11,13,19,22,37,41,43,48-49,55,57,59-61,67,79) & 20 & 25 \\
\hline Perda de relacionamento significativo $(1,6,7,22-24,37,43,45,48,52-54,63,65,71,74,76,81)$ & 18 & 22,5 \\
\hline Isolamento social| $(1,3,7,11,24,41,43,45,48-49,51,54,68,71,74,77,81,83)$ & 18 & 22,5 \\
\hline Perda da autonomia ${ }^{(1-2,13,24,29,36-38,43,45-46,48,52,78-79)}$ & 15 & 18,7 \\
\hline Aposentadoria ${ }^{(1,4,36,41,45-46,49,53,65,76,78-79,84)}$ & 13 & 16,2 \\
\hline Privação social ${ }^{(1,19,45-46,48,53,60,72,78-79)}$ & 10 & 12,5 \\
\hline Desvalorização social $\left.\right|^{(1,41,45-47,48,53,74,76,78-79)}$ & 10 & 12,5 \\
\hline Luto $^{(1,7,37,45-46,52-53,72,74,79)}$ & 10 & 12,5 \\
\hline Abandono $(1,22,45,48,52,78,79)$ & 7 & 8,7 \\
\hline Acesso a meios letais ${ }^{(1-2,22,37,47-48)}$ & 6 & 7,5 \\
\hline Violência psicológica $(46,48,52-53,55,77)$ & 6 & 7,5 \\
\hline Violência intrafamiliar $(46,48,53,55,60,79)$ & 6 & 7,5 \\
\hline Violência financeira ${ }^{(48,52-53,60,79)}$ & 5 & 6,2 \\
\hline Autonegligência ${ }^{(48,53)}$ & 2 & 2,5 \\
\hline
\end{tabular}

Fonte: Dados da pesquisa, 2015

\section{口 DISCUSSÃO}

Detectou-se a escassez de estudos na área da enfermagem voltados para o tema de suicídio de idosos. Nesse sentido, reforça-se também a escassez de pesquisas na América Latina, especificamente no contexto brasileiro.

A literatura enfatiza que, dentre os fatores psicológicos relacionados ao DE Risco de suicídio em idosos, os mais frequentes são os transtornos mentais. A exceção ocorre na
China, em que transtornos mentais em idosos que se suicidaram são $\operatorname{raros}^{(38)}$.

Estudo conduzido no Canadá(22) encontrou que idosos com depressão podem apresentar até sete vezes mais chance de cometer suicídio do que aqueles sem depressão. Estudo de coorte em Hong Kong ${ }^{(50)}$ revelou que a depressão esteve associada em 21,9\% dos casos de morte por suicídio em mulheres e 15,5\% em homens. Estudos no Brasil(52), na Austrália(25) e na Europa ${ }^{(82)}$ encontraram que a depressão assume múltiplas faces no sui- 
cídio de idosos, aparece como diagnóstico primário ou secundário, como sintoma associado a outras morbida$\operatorname{des}^{(12,20,22,25-26,31-32,36,38,59-60,64,67-69,71,73,77-78,81-82)}$ ou como reação a estressores sociais.

Estudo na Austrália ${ }^{(72)}$ revelou que $42 \%$ dos idosos que efetivaram o suicídio expressaram verbalmente o desejo de morrer, $25 \%$ tinham o pensamento de que a vida não valia mais a pena, $21 \%$ pensaram em tirar a vida e $7 \%$ fizeram plano suicida. Estudo no Brasil(45-47,52) revelou que os idosos verbalizaram o desejo de morrer, no entanto, os familiares e amigos não levaram a sério o problema ou não reconheceram essas pistas verbais como ideações suicidas ${ }^{(45-47,52)}$.

Ainda neste contexto, atenta-se para a inexistência do DE Ideação suicida na NANDA-I, o qual julgamos ser pertinente para pensar no planejamento e implementação de ações de enfermagem com foco na prevenção do suicídio.

Os idosos que morreram por suicídio possuíam histórico de tentativa de suicídio. Essa proporção variou de 11\% a $52 \%(20,29,31,33,62,69,72,73,81)$. Cerca de 11 a $27 \% \%^{(19-21,62,70,73,80)}$ desses idosos repetiram o ato com menos de um ano, utilizando métodos mais violentos ${ }^{(80)}$.

Os eventos traumáticos na infância estão associados ao suicídio. São experiências de vida marcadas por luto do pai na infância ${ }^{(80)}$, oferta insuficiente e inadequada de cuidado materno e paterno ${ }^{(67,75,78)}$, história de abuso sexual|(67) e violência intrafamiliar ${ }^{(84)}$. Esses acontecimentos podem refletir nos mecanismos de enfrentamento aos eventos estressantes na vida desses indivíduos.

No que tange aos fatores físicos, investigações sobre doenças crônico-degenerativas e suicídio de ido$\operatorname{sos}^{(22,26,28,31,39,58,60,68-69,82)}$ reportaram associações significativas do ato autodestrutivo com doenças cardiovasculares; doença pulmonar crônica; problemas de visão; diabetes mellitus; incontinência urinária; doença reumatoide e com doenças terminais, especialmente, câncer de próstata e pulmão no homem e câncer de mama na mulher. No Brasil(45-46,78-79), a impotência sexual esteve associada ao suicídio de homens.

Pessoas idosas diagnosticadas com sete ou mais doenças tiveram nove vezes maior risco de suicídio comparado àquelas que não possuíam diagnósticos ${ }^{(29)}$. Junto a essas doenças, a ocorrência de dores severas por mais de seis meses potencializa o risco de suicídio(28,32,68).

Dentre os fatores relacionados ao sentimento, os estu$\operatorname{dos}^{(7,12,14,22,39,43,59,78)}$ evidenciaram que esses idosos possuíam traços de personalidade para baixa consciência de si, instabilidade emocional, manifestada por sentimentos como ansiedade, melancolia e tristeza, pouca abertura para experiências, inflexibilidade e hostilidade. Estudo qualitativo ${ }^{(78)}$ evidenciou que os idosos eram intolerantes, controladores, rígidos e introvertidos. Tais características fazem com que os idosos tenham mais dificuldade em gerenciar os desafios do envelhecimento.

Mais da metade dos idosos que se suicidaram residiam sozinhos ${ }^{(1-3,3,6,11,13,21,23,37,41,43,48-49,55,57,59-61,67,79)}$. Sentimentos de solidão e falta de conectividade com a sociedade se configuram como preditivo para o suicídio(1,12,26,30,37,39,43,57,63,74,83). $\mathrm{O}$ apoio social inadequado amplia o horizonte do desamparo e os idosos se veem fragilizados por terem poucos amigos e familiares nos quais possam confiar (20,26,29-31,41,45,51-53,67-69,73,78-79). Estudo na Nova Zelândia ${ }^{(67)}$ assinalou que 27\% dos casos de suicídio poderiam ser evitados com apoio social adequado.

Os estudos evidenciam fatores situacionais e demográficos que não constam na NANDA-I: a aposentadoria em que o idoso perde a sua identidade social; sobrecarga econômica, em que o dever de ser o provedor da família o faz se sentir fracassado na tarefa; a privação social e a perda da autonomia, situações em que o idoso passa a residir com os filhos e perde seu espaço social; violência financeira, psicológica e intrafamiliar, em que o idoso é humilhado e desprezado pela família(1-2,37,39,41,43,45,48,52-53,55,62,67,73,78-79).

\section{CONCLUSÃO}

Aponta-se a existência de lacunas entre os fatores de risco para o DE Risco de suicídio, o que pode fragilizar a decisão do enfermeiro quanto à atribuição da presença ou ausência desse fenômeno. Sugere-se a inclusão de 23 novos fatores de risco para o DE Risco de suicídio: apatia; agitação; baixa autoestima; descuido com a medicação; incapacidade de pedir ajuda; incapacidade de expressar sentimentos; plano suicida; rigidez; incapacidade funcional; problemas visuais; tristeza; hostilidade; ansiedade; fracasso; frustração; infelicidade; desonra; frequentes visitas a médico com sintomas vagos; privação social; desvalorização social; violência psicológica; violência intrafamiliar; e violência financeira. Recomenda-se, para maior acurácia, a realização de novos estudos para o aprofundamento sobre essa temática e para a produção do conhecimento sobre esse diagnóstico. Ademais, é fundamental a realização da validação por especialistas e da validação clínica para confirmação desses resultados.

Limitações do estudo: a busca dos artigos foi executada por um único pesquisador, quando se orienta que seja feita aos pares; e a escassez de estudos brasileiros sobre risco de suicídio como um $D E$, o que restringiu a discussão dos dados e comparação com outros países.

Ao ampliar e inserir novos fatores de risco do DE Risco de suicídio, este estudo constrói caminhos para a prevenção do suicídio na população idosa, pois ao produzir no- 
vos conhecimentos em busca de decifrar esse fenômeno, cria novas possibilidades para o cuidado de enfermagem na promoção da saúde mental do idoso e da melhoria da qualidade de vida.

\section{口 REFERÊNCIAS}

1. Minayo MCS, Cavalcante FG. Suicide in elderly people: a literature review. Rev Saúde Pública. 2010;44(4):750-7. doi: http://dx.doi.org/10.1590/5003489102010000400020.

2. Conwell, Y. Suicide in later life: Challenges and priorities for prevention. Am J Prev Med. 2014;47(3S2):244-50. doi: http://dx.org/10.1016/j.amepre.2014.05.040.

3. American Association of Suicidology [Internet]. Elder suicide facts sheet [cited 2016 Sep 13]. Available from: http://www.sciencedaily.com.

4. O'Connell H, Chin A-V, Cunningham C, Lawlor BA. Recent developments: suicide in older people. BMJ. 2004;329(7471):895-9. doi: http://dx.doi.org/10.1136/ bmi.329.7471.895.

5. Pinto LW, Assis SG, Pires TO. Suicide mortality in people aged 60 and over in Brazilian municipalities between 1996 and 2007. Cienc Saude Coletiva. 2012;17(8):1963-72. doi: http://dx.doi.org/10.1590/5141381232012000800007.

6. Ciulla L, Nogueira EL, Silva Filho IG, Tres GL, Engroff P, Ciulla V, et al. Suicide risk in the elderly: data from Brazilian public health care program. J Affect Disord. 2014;152-154:513-6. doi: http://dx.doi.org/10.1016/j.jad.2013.05.090.

7. Conwell Y, Duberstein PR. Suicide in elders. Ann NY Acad Sci. 2001Apr;932(1):132-50. doi: http://dx.doi.org/10.1111/j.1749-6632.2001. tb05802.x.

8. North American Nursing Diagnosis Association. Diagnósticos de enfermagem da NANDA: definições e classificação 2015-2017. Porto Alegre: Artmed; 2015.

9. Ganong LH. Integrative reviews of nursing research. Res Nurs Health. 1987 Mar;10(1):1-11. doi: http://dx.doi.org10.1002/nur.4770100103.

10. Melnyk BM, Fineout-Overholt E. Making the case for evidence-based practice. In: Melnyk BM, Fineout-Overholt E. Evidence-based practice in nursing \& healthcare: a guide to best practice. Philadelphia: Lippincot Williams \& Wilkins; 2005. p. 3-24.

11. Hill RD, Gallagher D, Thompson LW, Ishida T. Hopelessness as a measure of suicide intent in the depressed elderly. Psychol Aging.1988;3(3):230-2. doi: http:// dx.doi.org/10.1037/0882-7974.3.3.230.

12. Duberstein PR. Openness to experience and completed suicide across the second half of life. Int Psychogeriatr. 1995; (2):183-98. doi: http://dx.doi.org/10.1017/ S1041610295001967.

13. Johnston M, Walker M. Suicide in the elderly: recognizing the signs. Gen Hosp Psychiatry. 1996;18(4):257-60. doi: http://dx.doi.org/10.1016/01638343(96)00039-4.

14. Forsell Y, Jorm AF, Winblad B. Suicidal thoughts and associated factors in an elderly population. Acta Psychiatr Scand. 1997;95(2):108-11. doi: http://dx.doi. org/10.1111/j.1600-0447.1997.tb00382.x.

15. Conwell Y. Management of suicidal behavior in the elderly. Psychiatr Clin North Am. 1997[cited 2016 Sep 13];20(3):667-83. Available from: http://www.sciencedirect.com/science/article/pii/S0193953X05703361/pdfft?md5=d40aeb c607a2a2da837805c5f5e73a3d\&pid=1-s2.0-S0193953X05703361-main.pdf

16. Seidlitz L, Conwell, Y, Duberstein P, Cox C. Denning, D. Emotion traits in older suicide attempters and non-attempters. J Affect Disord. 2001;66(2-3):123-31. doi: https://dx.doi.org/10.1016/50165-0327(00)00300-1.
17. Conner KR, Conwell Y, Duberstein PR. The validity of proxy-based data in suicide research: a study of patients 50 years of age and older who attempted suicide. II. Life events, social support, and suicidal behavior. Acta Psychiatr Scand. 2001;104(6):452-7. doi: http://dx.doi.org/10.1034/j.16000447.2001.00091.x.

18. Harwood D, Hawton K, Hope T, Jacoby R. Psychiatric disorder and personality factors associated with suicide in older people: a descriptive and casecontrol study. Int I Geriatr Psychiatry. 2001;16(2):155-65. doi: http://dx.doi. org/10.1002/1099-1166(200102) 16:2<155::AID-GPS289>3.0.c0;2-0.

19. Rubio A, Vestner AL, Stewart JM, Forbes NT, Conwell, Y, Cox C. Suicide and Alzheimer's pathology in the elderly: a case-control study. Biol Psychiatry. 2001;49(2):137-45. doi: https://dx.doi.org/10.1016/S0006-3223(00)009525.

20. Rubenowitz E, Waern M, Wilhelmsson K, Allebeck P. Life events and psychosocial factors in elderly suicides: a case control study. Psychol Med. 2001;31(7):1193202. doi: http://dx.doi.org/10.1017/S0033291701004457.

21. De Leo D, Padoani W, Scocco P, Lie D, Bille-Brahe U, Aresman E, et al. Attempted and completed suicide in older subjects: results from the WHO/EURO multicentre study of suicidal behaviour. Int I Geriatr Psychiatr. 2001;16(3):300-10. doi: http://dx.doi.org/10.1002/gps.337.

22. Quan H, Arboleda-Florez J, Fick GH, Stuart HL, Love EJ. Association between physical illness and suicide among the elderly. Soc Psychiatry Psychiatr Epidemiol. 2002[cited 2016 Sep 13];37(4):190-7. Available from: https://link. springer.com/content/pdf/10.1007\%2Fs001270200014.pdf.

23. Waern M, Runeson B, Allebeck P, Beskow J, Rubenowitz E, Skoog I, et al. Mental disorder in elderly suicides: a case-control study. Am J Psychiatry. 2002;159(3):450-5. doi: http://dx.doi.org/10.1176/appi.ajp.159.3.450.

24. Conwell Y, Duberstein PR, Caine ED. Risk factors for suicide in later life. Biol Psychiatry. 2002;52(3):193-204. doi: http://dx.doi.org/10.1016/500063223(02)01347-1.

25. Snowdon J, Baume P. A study of suicides of older people in Sydney. Int J Geriatr Psychiatry. 2002;17(3):261-9. doi: http://dx.doi.org/10.1002/gps.586.

26. Turvey C, Conwell Y, Jones MP, Phillips C, Simonsick E, Pearson JL, et al. Risk factors for late-life suicide: a prospective, community-based study. Am J Geriatr Psychiatry. 2002;10(4):398-406. doi: http://dx.doi.org/10.1097/00019442200207000-00006.

27. Charney D, Reynalds CF, Lewis L, Lebowitz BD, Sunderland, T, Alexopoulos GS, et al. Depression and bipolar support alliance consensus statement on the unmet needs in diagnosis and treatment of mood disorders in late life. Arch Ger Psychiatry. 2003;60(7):664-72. doi: http://dx.doi.org/10.1001/archpsyc.60.7.664.

28. Lebret S, Vaille EP, Mulliez A, Gerbaud L, Jalenques I. Elderly suicide attempters: characteristics and outcome. Int I Geriatr Psychiatry. 2006;21 (11):1052-9. doi: http://dx.doi.org/10.1002/gps. 1605.

29. Juurlink DN, Herrmann N, Szalai JP, Kopp A, Redelmeier DA. Medical illness and the risk of suicide in the elderly. Arch Intern Med. 2004;164(11):1179-84. doi: http://dx.doi.org/10.1001/archinte.164.11.1179.

30. Chou, KL. Reciprocal relationship between suicidal ideation and depression in Hong Kong elderly Chinese. Int J Geriatr Psychiatry. 2006;21(6):594-6. doi: http://dx.doi.org/10.1002/gps.1535.

31. Koponen $H$, Viilo K, Hakko H, Timonen M, Rochow VBM, Sarkioja T, et al. Rates and previous disease history in old age suicide. Int I Geriatr Psychiatry. 2007;22(1):38-46. doi: http://dx.doi.org/10.1002/gps. 1651.

32. Tadros $G$, Salib E. Elderly suicide in primary care. Int I Geriatr Psychiatry. 2007;22(8):750-6. doi: http://dx.doi.org/10.1002/gps.1734. 
33. Karvonen K, Rasanen P, Hakko H, Timonen M, Rochow VBM, Sarkioja T, et al. Suicide after hospitalization in the elderly: a population based study of suicides in Northern Finland between 1988-2003. Int J Geriatr Psychiatry. 2008;23(2):13541. doi: http://dx.doi.org/10.1002/gps.1853.

34. McGirr A, Renaud J, Bureau A, Sequin M, Lesage A, Turecki G. Impulsive-aggressive behaviours and completed suicide across the life cycle: a predisposition for younger age of suicide. Psychol Med. 2008;38(3):407-17. doi: http://dx.doi. org/10.1017/S0033291707001419.

35. Britton PC, Duberstein PR, Conner KR, Heisel MJ, Hirsch JK, Conwell, Y. Reasons for living, hopelessness, and suicide ideation among depressed adults 50 years or older. Am J Geriatr Psychiatry. 2008;16(9):736-41. doi: http://dx.doi. org/10.1097/JGP.0b013e31817b609a.

36. Chen CS, Yang MS, Yang MJ, Chang SJ, Chueh KH, Su YC, et al. Suicidal thoughts among elderly Taiwanese aboriginal women. Int J Geriatr Psychiatry. 2008;23(10):1001-6. doi: http://dx.doi.org/10.1002/gps.2024.

37. Conwell Y, Thompson C. Suicidal behavior in elders. Psychiatr Clin North Am. 2008;31(2):333-56. doi: http://dx.doi.org/10.1016/j.psc.2008.01.004.

38. Li X, Xiao Z, Xiao S. Suicide among the elderly in mainland China. Psychogeriatrics. 2009;9(2):62-6. doi: http://dx.doi.org/10.1111/j.14798301.2009.00269.x.

39. Conwell Y, Duberstein PR, Hirsch JK, Conner KR, Eberly S, Caine ED. Health status and suicide in the second half of life. Int J Geriatr Psychiatry. 2010;25(4):371-9. doi: http://dx.doi.org/10.1002/gps.2348.

40. Gibbs LM, Dombrovski AY, Morse J, Siegle GJ, Houck PR, Szanto, K. When the solution is part of the problem: problem solving in elderly suicide attempters. Int J Geriatr Psychiatry. 2009;24(12):1396-404. doi: http://dx.doi.org/10.1002/ gps.2276.

41. Kjolseth I, Ekeberg 0, Steihaug S. Elderly people who committed suicide: their contact with the health service. What did they expect, and what did they get? Aging Ment Health. 2010;14(8):938-46. doi: http://dx.doi.org/10.1080/13607 863.2010.501056.

42. Voshaar RCO, Kapur N, Bickley H, Williams A, Purandare, N. Suicide in later life: A comparison between cases with early-onset and late-onset depression. J Affect Disord. 2011;132(1-2):185-91. doi: http://dx.doi.org/10.1016/j. jad.2011.02.008.

43. Conwell Y, Van Orden K, Caine ED. Suicide in older adults. Psychiatr Clin North Am. 2011;34(2):451-68. doi: http://dx.doi.org/10.1016/j.psc.2011.02.002.

44. Levy TB, Barak Y, Sigler M, Aizenberg D. Suicide attempts and burden of physical illness among depressed elderly inpatients. Arch Gerontol Geriatr. 2011;52(1):115-7. doi: http://dx.doi.org/10.1016/j.archger.2010.02.012.

45. Sousa, GS, Silva RM, Figueiredo AEB, Minayo MCS, Vieira LJES. [Circumstances surrounding the suicide of elderly people]. Interface. 2014;18(49):389-402. doi: http://dx.doi.org/10.1590/1807-57622013.0241. Portuguese.

46. Cavalcante FG, Minayo MCS. Psychological and psychosocial autopsies of elderly people who died as a result of suicide in Brazil. Cienc Saude Coletiva. 2012;17(8):1943-54. doi: http://dx.doi.org/10.1590/S141381232012000800002 .

47. Richard-Devantoy S, Jollant F, Kefi Z, Turecki G, Olié JP, Annweiler C, et al. Deficit of cognitive inhibition in depressed elderly: a neurocognitive marker of suicidal risk. J Affect Disord. 2012;140(2):193-9. doi: http://dx.doi.org/10.1016/j. jad.2012.03.006.

48. Barrero SAP. Suicide risk factors among the elderly. Cienc Saude Coletiva. 2012;17(8):2011-6. doi: http://dx.doi.org/10.1590/S141381232012000800012 .
49. Martiello MA, Giacchi MV. Ecological study of isolation and suicide in Tuscany (Italy). Psychiatry Res. 2012;198(1):68-73. doi: http://dx.doi.org/10.1016/j. psychres.2011.11.013.

50. Sun WJ, Xu L, Chan WM, Lam TH, Schooling CM. Depressive symptoms and suicide in 56,000 older Chinese: a Hong Kong cohort study. Soc Psychiatry Psychiatr Epidemiol. 2012;47(4):505-14. doi: http://dx.doi.org/10.1007/s00127011-0362-z.

51. Jonson M, Skoog I, Marlow T, Fassberg MM, Waern, M. Anxiety symptoms and suicidal feelings in a population sample of 70-year-olds without dementia. Int Psychogeriatr. 2012;24(11):1865-71. doi: http://dx.doi.org/10.1017/ S1041610212000993.

52. Cavalcante FG, Minayo MCS, Mangas RMN. [Different aspects of depression in suicide among the elderly]. Cienc Saude Coletiva. 2013;18(10):2985-94. doi: http://dx.doi.org/10.1590/\$1413-81232013001000023. Portuguese.

53. Minayo MCS, Cavalcante FG. [A comprehensive study on suicide among older women in seven Brazilian cities]. Cad Saúde Pública. 2013;29(12):2405-15. doi: http://dx.doi.org//10.1590/0102-311X00048013. Portuguese.

54. Ojagbemi A, Oladeji B, Abiona T, Gureje 0. Suicidal behaviour in old age: results from the lbadan study of ageing. BMC Psychiatry. 2013;13:80. doi: http://dx.doi. org/10.1186/1471-244X-13-80.

55. Holm AL, Lyberg A, Berggren I, Cutcliffe J, Severinsson, E. Shadows from the past: the situated meaning of being suicidal among depressed older people living in the community. Crisis. 2014;35(4):253-60. doi: http://dx.doi. org/10.1027/0227-5910/a000259.

56. Cheung G, Casey J. Few older people in New Zealand who commit suicide receive specialist psychogeriatric services. Australas Psychiatry. 2014;22(4):3869. http://dx.doi.org/10.1177/1039856214537693.

57. Torresani S, Toffol E, Scocco P, Fanolla A. Suicide in elderly South Tyroleans in various residential settings at the time of death: a psychological autopsy study. Psychogeriatrics. 2014;14(2):101-9. doi: http://dx.doi.org/10.1111/psyg.12046.

58. Erlangsen A, Stenager E, Conwell Y. Physical diseases as predictors of suicide in older adults: a nationwide, register-based cohort study. Soc Psychiatry Psychiatr Epidemiol. 2015;50(9):1427-39. doi: http://dx.doi.org/10.1007/s00127-0151051-0.

59. Hung GC, Kwok CL, Yip PSF, Gunnell D, Chen, YY. Predicting suicide in older adults: a community-based cohort study in Taipei City, Taiwan. J Affect Disord. 2015;172:165-70. doi: http://dx.doi.org/10.1016/j.jad.2014.09.037.

60. Oude Voshaar RC, van der Veen DC, Hunt I, Kapur N. Suicide in late-life depression with and without comorbid anxiety disorders. Int I Geriatr Psychiatry. 2016;31(2):146-52. doi: http://dx.doi.org/10.1002/gps.4304.

61. Park JY, Han JW, Jeong H, Jeong HG, Kim TH, Yoon IY, et al. Suicidal behaviors in elderly Koreans: one-month-point prevalence and factors related to suicidality. J Affect Disord. 2013;150(1):77-83. doi: http://dx.doi.org/10.1016/j. jad.2013.02.025.

62. De Leo D, Draper BM, Snowdon J, Kolves K. Suicides in older adults: a case-control psychological autopsy study in Australia. J Psychiatr Res. 2013;47(7):980-8. doi: http://dx.doi.org/10.1016/j.jpsychires.2013.02.009.

63. Zeppegno P, Manzetti E, Valsesia R, Siliquini R, Ammirata G, De Donatis 0 , et al. Differences in suicide behaviour in the elderly: a study in two provinces of Northern Italy. Int J Geriatr Psychiatry. 2005;20(8):769-75. doi: http://dx.doi. org/10.1002/gps. 1354.

64. Inoue K, Tanil H, Fukunaga T, Abe S, Nishimura F, Kimura Y, et al. Psychosomatic tendency for suicide among the elderly in Mie Prefecture, Japan. Psychogeriatrics. 2007;7(2):44-8. doi: http://dx.doi.org/10.1111/j.1479-8301.2006.00159.x. 
65. Demirçin S, Akkoyun M, Yilmaz R, Gökdoğan MR. Suicide of elderly persons: towards a framework for prevention. Geriatr Gerontol Int. 2011;11(1):107-13. doi: http://dx.doi.org/10.1111/j.1447-0594.2010.00660.x.

66. Conwell Y, Raby WN, Caine ED. Suicide and aging. II: the psychobiological interface. Int Psychogeriatr. 1995;7(2):165-81. doi: http://dx.doi.org/10.1017/ S1041610295001955.

67. Beautrais AL. A case control study of suicide and attempted suicide in older adults. Suicide Life Threat Behav. 2002;32(1):1-9. doi: http://onlinelibrary. wiley.com/doi/10.1521/suli.32.1.1.22184/epdf.

68. Préville M, Hébert R, Boyer R, Bravo G, Seguin M. Physical health and mental disorder in elderly suicide: a case-control study. Aging Ment Health. 2005;9(6):576-84. doi: http://dx.doi.org/10.1080/13607860500192973.

69. Chi I, Yip PSF, Yu GKK, Halliday P. A study of elderly suicides in Hong Kong. Crisis. 1998;19(1):35-46. http://doi.org/10.1027/0227-5910.19.1.35.

70. Scocco P, De Leo D. One-year prevalence of death thoughts, suicide ideation and behaviours in an elderly population. Int J Geriatr Psychiatry. 2002;17(9):842-6. doi: http://onlinelibrary.wiley.com/doi/10.1002/gps.691/epdf.

71. Mills PD, Watts BV, Huh TJ, Boar S, Kemp J. Helping elderly patients to avoid suicide: a review of case reports from a National Veterans Affairs database. J Nerv Ment Dis. 2013;201(1):12-6. doi: http://dx.doi.org/10.1097/ NMD.0b013e31827ab29c.

72. Conner KR, Duberstein PR, Conwell Y. The validity of proxy-based data in suicide research: a study of patients 50 years of age and older who attempted suicide. I. Psychiatric diagnoses. Acta Psychiatr Scand. 2001;104(3):204-9. doi: http:// onlinelibrary.wiley.com/doi/10.1034/j.1600-0447.2001.00405.x/abstract.

73. Liu IC, Chiu CH. Case-control study of suicide attempts in the elderly. Int Psychogeriatr. 2009;21(5):896-902. doi: http://dx.doi.org/10.1017/ S1041610209990056.

74. Klinger J. Suicide among seniors. Australas J Ageing. 1999;1(3):114-8. doi: http://dx.doi.org/10.1111/j.1741-6612.1999.tb00110.x

75. Kageyama T. Views on suicide among middle-aged and elderly populations in Japan: their association with demographic variables and feeling shame in seeking help. Psychiatry Clin Neurosci. 2012;66(2):105-12. doi: http://dx.doi. org/10.1111/j.1440-1819.2011.02313.x.
76. Køjlseth I, Ekeberg Ø, Steihaug S. Why do they become vulnerable when faced with the challenges of old age? Elderly people who committed suicide, described by those who knew them. Int Psychogeriatr. 2009;21(5):903-12. doi: http://dx.doi.org/10.1017/S1041610209990342.

77. Duberstein PR, Conwell Y, Conner KR, Eberly S, Caine ED. Suicide at 50 years of age and older: perceived physical illness, family discord and financial strain. Psychol Med. 2004;34(1):137-46. doi: http://dx.doi.org/10.1017/ S0033291703008584.

78. Meneghel SN, Gutierrez DMD, Silva RMS, Grubits S, Hesler LZ, Ceccon RF. Suicide in the elderly from a gender perspective. Cienc Saude Coletiva. 2012;17(8):198392. doi: http://dx.doi.org/10.1590/S1413-81232012000800009.

79. Minayo MCS, Meneghel SN, Cavalcante FG. Suicide of elderly men in Brazil. Cienc Saude Coletiva. 2012;17(10):2665-74. doi: http://dx.doi.org/10.1590/ S1413-81232012001000016.

80. De Leo D, Padoani W, Lonnqvist J, Kerkhof AJ, Bille-Brahe U, Michel K, et al. Repetition of suicidal behaviour in elderly Europeans: a prospective longitudinal study. J Affect Disord. 2002;72(3):291-5. https://dx.doi.org/10.1016/S01650327(01)00454-2.

81. Erlangsen A, VachW, Jeune B. The effect of hospitalization with medical illnesses on the suicide risk in the oldest old: a population-based register study. J Am Geriatr Soc. 2005;53(5):771-6. doi: http://dx.doi.org/10.1111/j.1532-5415.2005.53256.x.

82. Waern M, Rubenowitz E, Runeson B, Skoog I, Wilhelmson K, Allebeck P. Burden of illness and suicide in elderly people: case-control study. BMJ. 2002;324(7350):1355. doi: https://doi.org/10.1136/bmj.324.7350.1355.

83. Yur yev A, Leppik L, Tooding LM, Sisask M, Värnik P, Wu J, et al. Social inclusion affects elderly suicide mortality. Int Psychogeriatr. 2010;22(8):1337-43. doi: http://dx.doi.org/10.1017/S1041610210001614.

84. Sorock GS, Chen L-H, Gonzalgo SR, Baker SP. Alcohol-drinking history and fatal injury in older adults. Alcohol. 2006;40(3):193-9. doi: http://dx.doi. org/10.1016/j.alcohol.2007.01.002.

85. Shah A, Bhat R. The relationship between elderly suicide rates and mental health funding, service provision and national policy: a cross-national study. Int Psychogeriatr. 2008;20(3):605-15. doi: http://dx.doi.org/10.1017/ S1041610207006552.

\section{- Autor correspondente:}

Girliani Silva de Sousa

Email: girlianis@gmail.com
Recebido: 17.06.2017

Aprovado: 06.10.2017 